\title{
Effects of Teriparatide and Aerobic Exercise on Lumbar Spine Microstructure in Ovariectomized and Tail-Suspended Rats
}

\author{
Chiaki Sato, Naohisa Miyakoshi*, Yuji Kasukawa, Koji Nozaka, Hiroyuki Tsuchie, \\ Itsuki Nagahata, Yusuke Yuasa, Kazunobu Abe, Hikaru Saito, Ryo Shoji, Yoichi Shimada \\ Department of Orthopedic Surgery, Akita University Graduate School of Medicine, Akita, Japan \\ Email: chakky.5996@gmail.com, ^miyakosh@doc.med.akita-u.ac.jp, kasukawa@doc.med.akita-u.ac.jp, \\ nozakak@med.akita-u.ac.jp, tsuchie@doc.med.akita-u.ac.jp, nagahata.itsuki.1986@gmail.com, yuasayusuke@gmail.com, \\ kabe@med.akita-u.ac.jp, saitouhikaru@med.akita-u.ac.jp, shojiryo613@med.akita-u.ac.jp, seikei@doc.med.akita-u.ac.jp
}

How to cite this paper: Sato, C., Miyakoshi, N., Kasukawa, Y., Nozaka, K., Tsuchie, H., Nagahata, I., Yuasa, Y., Abe, K., Saito, H., Shoji, R. and Shimada, Y. (2021) Effects of Teriparatide and Aerobic Exercise on Lumbar Spine Microstructure in Ovariectomized and Tail-Suspended Rats. Open Journal of Orthopedics, 11, 1-11. https://doi.org/10.4236/ojo.2021.111001

Received: November 30, 2020

Accepted: January 9, 2021

Published: January 12, 2021

Copyright $\odot 2021$ by author(s) and Scientific Research Publishing Inc. This work is licensed under the Creative Commons Attribution International License (CC BY 4.0).

http://creativecommons.org/licenses/by/4.0/

\begin{abstract}
Osteoporosis is an increasingly prevalent malady of the elderly that is associated with bone fragility and increased risk of fractures. Osteoporosis treatments focus on restoring bone strength and quality. Teriparatide (TPTD) is a therapeutic agent that has been shown to increase bone strength by improving the volume and connectivity of trabecular bone. Exercise is also known to have pro-osteogenic effects. Here we used a rat model of severe osteoporosis (ovariectomized and tail-suspension) to evaluate the effects of TPTD, exercise and a combination of TPTD and exercise on the microstructure of trabecular bone. TPTD mono-therapy and TPTD combined with exercise treatment significantly increased bone mineral density (BMD) in the whole body. Micro-computed tomography analysis revealed that a combination of exercise and TPTD treatment significantly decreased bone surface to volume and trabecular separation compared with those of the control and exercise groups. Node-strut analysis indicated that exercise or TPTD alone did not affect trabecular bone connectivity. However, the combination of exercise and TPTD treatment significantly decreased measures of trabecular bone connectivity (node number) that are consistent with a transition from rod-like to plate-like of trabecular bone microstructures. The combination treatment with exercise and TPTD improved microstructure of trabecular bone in the OVX and tail-suspended rats. These results indicate that combining exercise with TPTD represents a viable means to improve cancellous bone strength in osteoporosis populations.
\end{abstract}

\section{Keywords}

Teriparatide, Aerobic Exercise, Micro Structure, Cancellous Bone 


\section{Introduction}

Bone fragility due to osteoporosis is associated with an increased risk of fractures in the elderly [1]. The number of elderly individuals is increasing rapidly, thus effective treatments for osteoporosis are very important to reducing the risk of fragility fractures in the elderly. One of the goals of osteoporosis treatment is to prevent fragility fractures by improving bone strength, as defined by bone mineral density (BMD) and bone quality [2]. Bone quality consists of two factors: structural characteristics and material characteristics. The structural characteristics of cancellous bone are defined by several factors including the connectivity and microstructure of trabecular bone such as plate-like or rod-like [3]. It has been reported that the microarchitecture of trabecular bone is a structural characteristic that plays a significant role in cancellous bone strength and determines its biomechanical properties [4]. Thus, it is vital to investigate the effects of osteoporosis treatments on the microstructure of trabecular bone using a model of severe osteoporosis.

Teriparatide (TPTD) is a therapeutic agent used to treat osteoporosis that has been shown to increase bone strength by improving the connectivity of trabecular bone as well as increasing bone volume [5] and BMD [6] in a rat model of osteoporosis. However, we previously reported that TPTD was unable to restore the connectivity of trabecular bone and bone strength in a severe osteoporosis model of ovariectomized (OVX) and sciatic neurectomized (NX) rats [5]. Therefore, TPTD alone may not be able to restore the connectivity and microstructure of trabecular bone in severe osteoporosis, and additional treatment(s) may be required to augment the effects of TPTD on trabecular bone in such cases.

Exercise therapy is a potential candidate for use in combination therapy. Exercise increases systemic parathyroid hormone (PTH) levels depending on the type, intensity, and duration of exercise [7] [8] [9], and has a dynamic loading effect on bone tissue. It has been reported that loading and high-dose intermittent PTH administration revealed synergistic effects on cortical bone volume and cortical bone formation of the proximal tibia and distal ulna in mice [10] [11].

In view of this, aerobic exercise therapy may enhance the effect of TPTD on the microstructure of trabecular bone. However, the effects of combined exercise therapy and TPTD on the connectivity and microstructure of trabecular bone remain unclear. Therefore, we investigated the effects of aerobic exercise therapy and TPTD in combination on trabecular bone connectivity and microstructure in severe osteoporosis model rats (OVX + tail suspension).

\section{Materials and Methods}

\subsection{Animals and Experimental Protocol}

Seven-month-old female Wistar rats (Japan SLC, Shizuoka, Japan) were housed in a controlled environment (temperature $23^{\circ} \mathrm{C} \pm 2{ }^{\circ} \mathrm{C}$, humidity $40 \% \pm 20 \%$ ) 
with a 12-h light/dark cycle.

An ovariectomy was performed under general anesthesia at 7 months of age as a model of estrogen deficiency. General anesthesia was induced by intraperitoneal injection of xylazine hydrochloride (Sederac; Nippon Zenyaku Kogyo, Fukushima, Japan) and ketamine hydrochloride (Ketalar; Daiichi Sankyo Propharma, Tokyo, Japan). Rats were tail-suspended for 4 weeks to create hindlimb unloading at 2 weeks after OVX. Finally, rats were randomly assigned to one of the following four groups $(\mathrm{n}=5$ /group $)$ at six weeks after OVX: Cont group, control group administered TPTD vehicle without low-intensity aerobic exercise training; Exe group, exercise group subjected to low-intensity aerobic exercise using a treadmill; TPTD group, teriparatide group administered TPTD at 30 $\mu \mathrm{g} / \mathrm{kg}$; and Comb group, combined group administered TPTD in combination with low-intensity aerobic exercise. After 8 weeks of treatment, the following parameters were evaluated. The animals were anesthetized by intraperitoneal injection of xylazine hydrochloride and ketamine hydrochloride for measurement of whole body BMD. Rats were euthanized by injection of sodium pentobarbital $(150 \mathrm{mg} / \mathrm{kg}$ body weight) (Dainippon Sumitomo Pharma, Osaka, Japan), and the lumbar vertebrae were harvested for evaluation of the trabecular connectivity and the micro-structure of trabecular bone using the micro-computed tomography.

The protocols for all animal experiments were approved in advance by the Animal Research Committee of Akita University Graduate School of Medicine, and all subsequent animal experiments adhered to the "Guidelines for Animal Experimentation" of Akita University Graduate School of Medicine (approval number: a-1-2935, which was approved in August/03/2017).

\subsection{Tail-Suspension Model}

Hindlimb unloading was performed as described previously [12]. The animals were suspended by the tail to maintain the rat at a head-down tilt of $30^{\circ}$ with the hindlimbs elevated above the floor of the cage for 28 days. The rats were allowed a $360^{\circ}$ range of motion to facilitate free movement about the cage.

\subsection{TPTD Administration and Treadmill Exercise}

TPTD (Asahi Kasei Pharma, Tokyo, Japan) was dissolved in sterile saline containing $0.1 \%$ rat serum albumin, and a dose of $30 \mu \mathrm{g} / \mathrm{kg}$ body weight was administered subcutaneously three times per week for 8 weeks based on a previously reported protocol [6] [13].

Low-intensity treadmill exercise was applied at a speed of $10 \mathrm{~m} / \mathrm{min}$ at a $5 \%$ incline for $60 \mathrm{~min} /$ day, 5 days/week for 8 weeks (MK-680; Muromachi Kikai, Tokyo, Japan). The treadmill exercise conditions were determined based on our previous study [14]. All rats completed the treadmill exercise throughout the entire experimental period.

\subsection{Tissue Preparation}

After the animals were sacrificed, the lumbar vertebrae (L2-4) were excised and 
fixed in 10\% neutral-buffered formalin (Wako Chemical Industries, Osaka, Japan) prior to micro-computed tomography measurements.

\subsection{BMD Measurement}

BMD of the whole body was measured using dual-energy X-ray absorptiometry (DXA) (QDR-4500 Delphi; Hologic, Bedford, MA, USA). Each region was scanned in "small animal" mode with the "regional high-resolution" scan option.

\subsection{Micro-Computed Tomography Measurement}

The excised lumbar spines from the four groups treated for 8 weeks ( $\mathrm{n}=5$ each) were secured in a sample holder. Micro-computed tomography was performed using CosmoScan GX II (Rigaku Corporation, Tokyo, Japan) according to the manufacturer's instructions, with an isotropic voxel size of $36 \mu \mathrm{m}$, energy of 90 $\mathrm{kVp}$, and current of $88 \mu \mathrm{A}$. Captured images were rendered using TRI/3D BON (Ratoc System Engineering Co., Ltd., Tokyo, Japan) software. Evaluation of trabecular bone volume and structure was performed based on bone surface/bone volume (BS/BV $[1 / \mathrm{mm}])$ and trabecular separation $(\mathrm{Tb} . \mathrm{Sp}[\mu \mathrm{m}])$. To evaluate trabecular bone connectivity, Node-strut analysis parameters including the number of nodes (N.Nd/TV $\left.\left[1 / \mathrm{mm}^{3}\right]\right)$, number of terminus (N.Tm/TV $\left.\left[1 / \mathrm{mm}^{3}\right]\right)$, node to node length $\left(\mathrm{NdNd} / \mathrm{TV}\left[1 / \mathrm{mm}^{3}\right]\right)$, node to terminus length $(\mathrm{NdTm} / \mathrm{TV}$ $\left.\left[1 / \mathrm{mm}^{3}\right]\right)$, and terminus to terminus length $\left(\mathrm{TmTm} / \mathrm{TV}\left[1 / \mathrm{mm}^{3}\right]\right)$ were measured. In addition, to analyze the microstructure of trabecular bone, trabecular bone pattern factor (TBPf $[1 / \mathrm{mm}]$ ), and structure model index (SMI) were also measured. TBPf expresses the ratio of convex to concave trabecular surfaces, thus indicating the level of trabecular connectivity [3]. SMI is an index of trabecular structure and expresses the ratio of plate-like to rod-like structures [15].

\subsection{Statistical Analysis}

All data are expressed as mean \pm standard deviation (SD). A Kolmogorov-Smirnov test showed that all data were normally distributed. Differences between groups were evaluated using one-way analysis of variance (ANOVA) and post hoc analysis was conducted using Scheffe's method. All statistical analyses were performed using the Statistical Package for the Biosciences (SPBS) version 9.6 (Akita University, Akita, Japan) [16]. Values of $p<0.05$ were considered significant.

\section{Results}

\subsection{Body Weight}

Body weights at the beginning of the experiment were not significantly different among the groups. The body weight (mean \pm standard deviation) was significantly lower in the Exe $(260.6 \pm 12.7 \mathrm{~g})$ and Comb groups $(260.6 \pm 14.1 \mathrm{~g})$ than in the Cont group $(284.8 \pm 15.5 \mathrm{~g})(\mathrm{p}<0.05)$ after 8 weeks of treatment. 


\subsection{BMD of Whole Body}

The BMDs at 8 weeks after treatment are shown in Figure 1. The BMD of the whole body was significantly increased with TPTD treatment $(\mathrm{p}<0.05)$ and combined treatment with exercise and TPTD $(\mathrm{p}<0.01)$ compared with that of the control group.

\subsection{Micro-Computed Tomography}

Exercise did not affect any of the parameters regarding bone volume and trabecular bone structure. Combined treatment with exercise and TPTD significantly decreased BS/BV (Figure 2(a)) and Tp.Sp (Figure 2(b)) compared with the control and exercise groups $(\mathrm{p}<0.05-\mathrm{p}<0.01)$. Axial images of the lumbar spine obtained by micro-CT showed that the trabecular bones were thicker in the Comb group (Figure 3(d)) than in the Cont group (Figure 3(a)).

Table 1 shows the results of Node-strut analysis and microstructure determination using TBPf and SMI. Exercise did not affect the Node-strut analysis parameters and TBPf and SMI. TPTD monotherapy significantly improved TBPf compared with the control group $(\mathrm{p}<0.05)$. Combined treatment with exercise and TPTD significantly decreased the node number (N.Nd/TV) compared with the control, exercise, and TPTD mono-therapy groups $(\mathrm{p}<0.05-\mathrm{p}<$ 0.01 ), and significantly decreased the node to node length ( $\mathrm{NdNd} / \mathrm{TV}$ ) compared with the control group. In addition, the combined treatment showed significantly improved TBPf and SMI compared with the control, exercise, and TPTD mono-therapy groups $(\mathrm{p}<0.05-\mathrm{p}<0.01)$.

Table 1. Micro-computed tomography of the lumbar spine, Node-Strut analysis and microstructure.

\begin{tabular}{cccccc}
\hline & Cont & Exe & TPTD & Comb & ANOVA \\
\hline Node-Strut analysis & & & & & \\
N.Nd/TV $\left(1 / \mathrm{mm}^{3}\right)$ & $27.7 \pm 2.1$ & $22.2 \pm 2.4$ & $22.4 \pm 4.6$ & $14.7 \pm 4.0^{\mathrm{a} b c}$ & $<0.0001$ \\
N.Tm/TV $\left(1 / \mathrm{mm}^{3}\right)$ & $0.7 \pm 0.4$ & $0.4 \pm 0.1$ & $0.6 \pm 0.2$ & $0.4 \pm 0.2$ & 0.0490 \\
NdNd/TV $\left(1 / \mathrm{mm}^{3}\right)$ & $13.9 \pm 1.2$ & $12.8 \pm 0.8$ & $12.6 \pm 1.2$ & $10.5 \pm 2.4^{\mathrm{a}}$ & 0.0106 \\
NdTm/TV $\left(1 / \mathrm{mm}^{3}\right)$ & $0.2 \pm 0.04$ & $0.1 \pm 0.08$ & $0.2 \pm 0.13$ & $0.15 \pm 0.05$ & 0.2605 \\
TmTm/TV $\left(1 / \mathrm{mm}^{3}\right)$ & $0.03 \pm 0.03$ & $0.01 \pm 0.01$ & $0.02 \pm 0.01$ & $0.01 \pm 0.01$ & 0.3628 \\
Micro structure & & & & & $<0.0001$ \\
TBPf & $-0.2 \pm 1.6$ & $-3.1 \pm 1.9$ & $-3.4 \pm 1.11^{\mathrm{a}}$ & $-6.1 \pm 1.3^{\mathrm{a}^{\mathrm{a} b c}}$ & $<0.0001$ \\
\hline
\end{tabular}

$\mathrm{n}=5$ per group. Values represent the means \pm SD. Cont: control group, administered vehicle without aerobic exercise, Exe: exercise group, performed aerobic exercise training, TPTD. teriparatide group, administered teriparatide at $30 \mu \mathrm{g} / \mathrm{kg}$, three times/week, Comb: combined group, administered teriparatide and performed aerobic exercise training, $A N O V A$ : analysis of variance. N.Nd/TV: number of nodes, N.Tm/TV: number of terminus, $N d N d / T V$ : node to node length, $N d T m / T V$ : node to terminus length, $T m T m / T V$ : terminus to terminus length, TBPf: trabecular bone pattern factor, $S M I$ : structure model index. ${ }^{a} \mathrm{p}<0.05$, ${ }^{a} \mathrm{p}<0.01$ vs. Cont group with Scheffe's method; ' $\mathrm{p}<0.05$, b' $\mathrm{p}<0.01$ vs. Exe group with Scheffe's method; ' $\mathrm{p}$ $<0.05,{ }^{\text {, }} \mathrm{p}<0.01$ vs. TPTD group with Scheffe's method. 


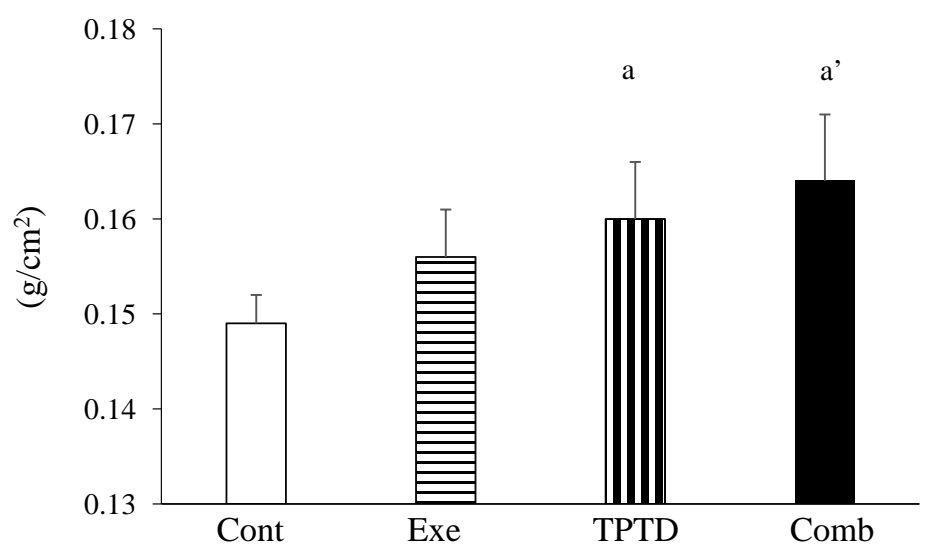

Figure 1. Bone mineral density in the whole body. Values represent the mean $\pm \mathrm{SD}$. ${ }^{\mathrm{a}} \mathrm{p}<$ $0.05,{ }^{a} \mathrm{p}<0.01$ vs. Cont group using Scheffe's method. Cont: control group, administered vehicle without aerobic exercise; Exe: exercise group, performed aerobic exercise training; TPTD: teriparatide group, administered teriparatide at $30 \mu \mathrm{g} / \mathrm{kg}$, three times/week; Comb: combined group, administered teriparatide and performed aerobic exercise training.

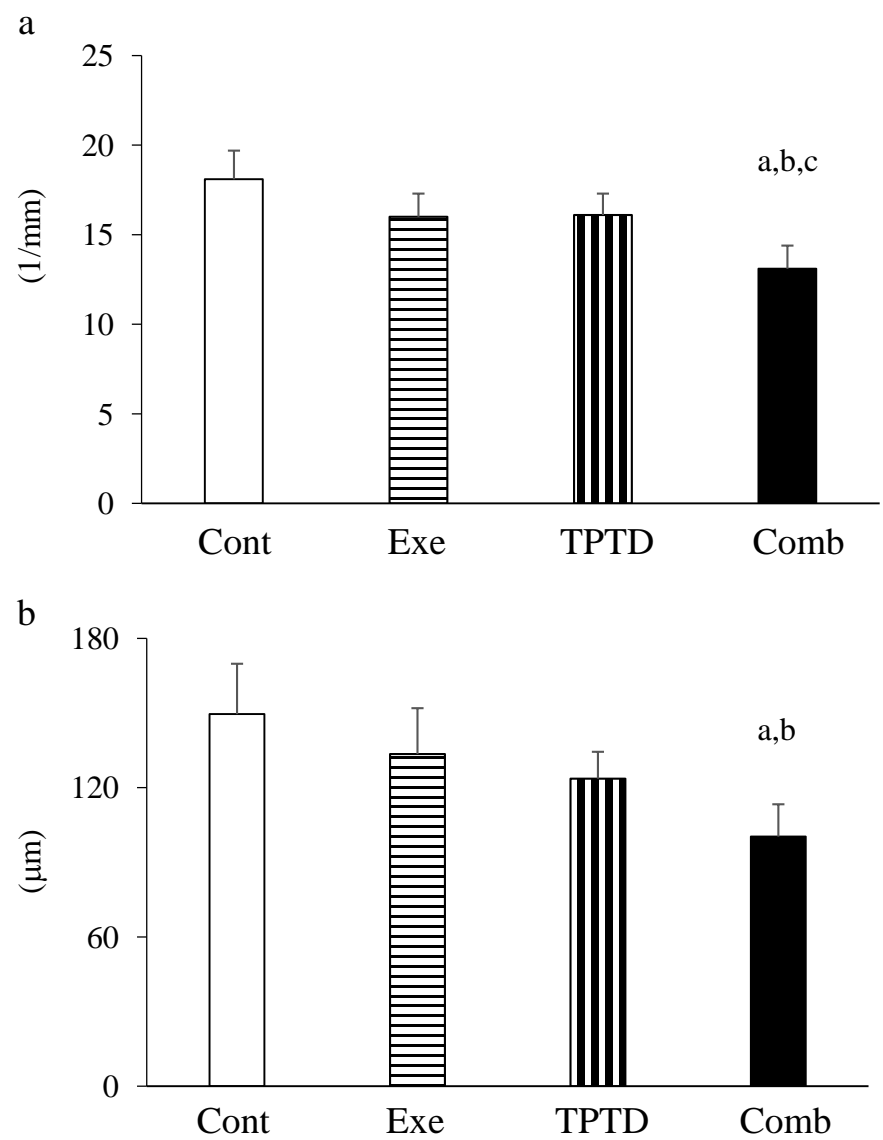

Figure 2. Lumbar spine bone volume (a: BS/BV) and trabecular bone structure (b: Tb.Sp) by micro-computed tomography. Values represent the mean $\pm \mathrm{SD}$. ${ }^{\mathrm{a}} \mathrm{p}<0.01$ vs. Cont group, ${ }^{b} \mathrm{p}<0.05$ vs. Exe group, ${ }^{c} \mathrm{p}<0.05$ vs. TPTD group using Scheffe's method. BS/BV: bone surface/bone volume; Tb.Sp: trabecular separation; Cont: control group, administered vehicle without aerobic exercise; Exe: exercise group, performed aerobic exercise training; TPTD: teriparatide group, administered teriparatide at $30 \mu \mathrm{g} / \mathrm{kg}$, three times/week; Comb: combined group, administered teriparatide and performed aerobic exercise training. 

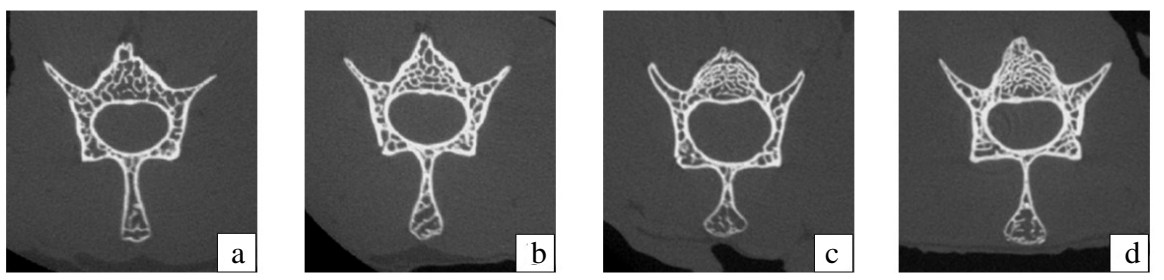

Figure 3. Axial images of the lumbar spine by micro-CT. Cont (control) group, administered vehicle without aerobic exercise (a); Exe (exercise) group, performed aerobic exercise training (b); TPTD (teriparatide) group, administered teriparatide at 30 $\mu \mathrm{g} / \mathrm{kg}$, three times/week (c); and Comb (combined) group, administered teriparatide and performed aerobic exercise training $(\mathrm{d})$.

\section{Discussion}

In this study, the combined treatment with aerobic exercise (treadmill) and TPTD increased the BMD of the whole body, and improved the microstructure of the trabecular bone from rod-like to plate-like according to TBPf and SMI, parameters related to the mechanical property of cancellous bone. In contrast, the parameters related to the connectivity in trabecular bone were not improved by the combined treatment with aerobic exercise and TPTD in the OVX and tail-suspended rats. Moreover, mono-therapy with aerobic exercise or TPTD did not affect these parameters in this study.

The parameters TBPf and SMI reflect the microstructure of trabecular bone and correlate with bone strength. SMI has been shown to deteriorate in OVX rats [15] and is elevated in subjects with a history of osteoporotic fragility fractures compared with those with no such history [17]. Thus, it is very important to improve trabecular bone microstructure parameters (i.e. TBPf and SMI). Intermittent administration of PTH [18], [19] and three times weekly administration of TPTD [6] have been shown to improve TBPf and SMI in OVX rats, and TPTD improves these parameters in patients with primary osteoporosis [20]. However, TPTD mono-therapy did not recover TBPf and SMI in the severe osteoporosis model (OVX + tail-suspension) rats in this study. Although it is necessary to investigate the effect of other doses or durations of TPTD treatment on these parameters of trabecular microstructure, the combined treatment with TPTD and exercise improved these parameters in this model. The combined treatment with TPTD and exercise is suggested to be another option to improve trabecular bone microstructure, and by extension, the strength of cancellous bone.

The parameters describing trabecular bone connectivity, such as N.Nd/TV and $\mathrm{NdNd} / \mathrm{TV}$, were not improved by the combined treatment with aerobic exercise and TPTD in the severe osteoporosis model rats in this study. Our previous study demonstrated that intermittent administration of PTH increased the connectivity of trabecular bone in OVX + NX rats, according to a two-dimensional Node-strut analysis [5]. However, another study reported that intermittent administration of PTH thickened the trabecular plates and did not increase trabecular connectivity in OVX rats by three-dimensional high-resolution microsco- 
py [21]. The absence of an effect on trabecular bone connectivity might be caused by an increase in the width of the trabecular bone and changes in trabecular structure from rod-shaped to plate-shaped.

The exercise used in this study is considered to be a model of aerobic exercise, such as walking and running on a treadmill. Weight-bearing exercise, such as running and jumping, are thought to have high osteogenic potential [22] [23] [24]. However, level and downhill running exercise failed to show significant effects on structural and microarchitectural changes of trabecular bone in growing rats [25]. Moreover, reloading subsequent to mechanical unloading did not recover the bone volume of trabecular bone and trabecular structures such as Tb.Th in aged rats [26]. Nevertheless, exercise therapy with a treadmill in combination with TPTD significantly improved trabecular microstructure parameters compared to the respective monotherapy in this study. The mechanism likely involves the occurrence of adaptation during exercise due to dynamic loading as well as PTH release, and PTH signaling during exercise contributes to improvements in the structural-level mechanical properties of cortical bone [27].

In clinical practice, we used OVX and tail-suspended osteoporosis rats as a model for elderly osteoporosis patients who spend large amounts of time in bed and have decreased activity. TPTD is a highly effective drug and shows a clear effect of increasing BMD and suppressing the occurrence of fractures. However, for elderly people with decreased activity and advanced osteoporosis, treatment with TPTD alone may not restore bone strength and fractures. In such situations, the use of TPTD in combination with exercise, even minimal activity such as standing or walking, appears to be useful for improving the fine structure of trabecular bone and eventually improving cancellous bone strength.

However, there are several limitations in this study. First, evaluation of the treatment effect on the microstructure of trabecular bone was performed at only one time point ( 8 weeks after treatment). Second, a single dose of TPTD and a fixed condition of treadmill exercise were used in this study. A longer treatment duration, higher TPTD dose, and more robust treadmill exercise might reveal significant effects on the microstructure of trabecular bone even with mono-therapy.

\section{Conclusion}

The present study investigated the effects of TPTD, exercise and a combination of TPTD and exercise on the microstructure of trabecular bone in the lumbar spine of rats model of severe osteoporosis (ovariectomized and tail-suspension). The combined treatment with aerobic exercise and TPTD recovered the microstructure of trabecular bone, such as TBPf and SMI, but not the connectivity, and showed significant effects on BMD and bone volume as well as trabecular thickness. These results indicate that combining exercise with TPTD represents a viable means to improve cancellous bone strength in elderly osteoporosis populations with decrease in their activity. 


\section{Authorship Contribution Statement}

Chiaki Sato: Investigation, Validation, Visualization, Writing original draft. Itsuki Nagahata: Investigation, Validation. Yusuke Yuasa: Investigation, Validation. Kazunobu Abe: Investigation. Hikaru Saito: Investigation. Ryo Shoji: Investigation. Hiroyuki Tsuchie: Investigation, Formal analysis. Koji Nozaka: Investigation, Formal analysis. Yuji Kasukawa: Conceptualization, Methodology, Writing review \& editing. Naohisa Miyakoshi: Conceptualization, Methodology, Project administration. Writing review \& editing. Yoichi Shimada: Conceptualization, Funding acquisition, Supervision.

\section{Acknowledgements}

The authors would like to thank Asahi Kasei Pharma Corporation for providing the TPTD and Ms. Matsuzawa and Ms. Kudo for their support of our experiments.

\section{Funding}

This research did not receive any specific grant from funding agencies in the public, commercial, or not-for-profit sectors.

\section{Ethical Approval}

The protocols for all animal experiments were approved in advance by the Animal Research Committee of our institute, and all subsequent animal experiments adhered to the "Guidelines for Animal Experimentation" of our university (approval number: a-1-2935).

\section{Conflicts of Interest}

Naohisa Miyakoshi has received payments for lectures from Asahi Kasei Pharma Corporation. The other authors declare that they have no conflicts of interest.

\section{References}

[1] Edwards, M.H., Dennison, E.M., Aihie Sayer, A., Fielding, R. and Cooper, C. (2015) Osteoporosis and Sarcopenia in Older Age. Bone, 80, 126-130. https://doi.org/10.1016/j.bone.2015.04.016

[2] NIH Consensus Development Panel on Osteoporosis Prevention, Diagnosis, and Therapy (2001) Osteoporosis Prevention, Diagnosis, and Therapy. JAMA, 285, 785-795. https://doi.org/10.1001/jama.285.6.785

[3] Hahn, M., Vogel, M., Pompesius-Kempa, M. and Delling, G. (1992) Trabecular Bone Pattern Factor: A New Parameter for Simple Quantification of Bone Microarchitecture. Bone, 13, 327-330. https://doi.org/10.1016/8756-3282(92)90078-B

[4] Ulrich, D., van Rietbergen, B., Laib, A. and Rüegsegger, P. (1999) The Ability of Three-Dimensional Structural Indices to Reflect Mechanical Aspects of Trabecular Bone. Bone, 25, 55-60. https://doi.org/10.1016/S8756-3282(99)00098-8

[5] Kasukawa, Y., Miyakoshi, N., Itoi, E., Tsuchida, T., Tamura, Y., Kudo, T., Suzuki, K., Seki, A. and Sato, K. (2004) Effects of h-PTH on Cancellous Bone Mass, Connectivity, 
and Bone Strength in Ovariectomized Rats with and without Sciatic-Neurectomy. Journal of Orthopaedic Research, 22, 457-464. https://doi.org/10.1016/j.orthres.2003.08.017

[6] Takao-Kawabata, R., Isogai, Y., Takakura, A., Shimazu, Y., Sugimoto, E., Nakazono, O., Ikegaki, I., Kuriyama, H., Tanaka, S., Oda, H. and Ishizuka, T. (2015) Three-Times-Weekly Administration of Teriparatide Improves Vertebral and Peripheral Bone Density, Microarchitecture, and Mechanical Properties without Accelerating Bone Resorption in Ovariectomized Rats. Calcified Tissue International, 97, 156-168. https://doi.org/10.1007/s00223-015-9998-0

[7] Brahm, H., Piehl-Aulin, K. and Ljunghall, S. (1997) Bone Metabolism during Exercise and Recovery: The Influence of Plasma Volume and Physical Fitness. Calcified Tissue International, 61, 192-198. https://doi.org/10.1007/s002239900322

[8] Iwamoto, J., Shimamura, C., Takeda, T., Abe, H., Ichimura, S., Sato, Y. and Toyama, Y. (2004) Effects of Treadmill Exercise on Bone Mass, Bone Metabolism, and Calciotropic Hormones in Young Growing Rats. Journal of Bone and Mineral Metabolism, 22, 26-31. https://doi.org/10.1007/s00774-003-0443-5

[9] Scott, J.P.R., Sale, C., Greeves, J.P., Casey, A., Dutton, J. and Fraser, W.D. (2011) The Role of Exercise Intensity in the Bone Metabolic Response to an Acute Bout of Weight-Bearing Exercise. Journal of Applied Physiology, 110, 423-432.

https://doi.org/10.1152/japplphysiol.00764.2010

[10] Sugiyama, T., Saxon, L.K., Zaman, G., Moustafa, A., Sunters, A., Price, J.S. and Lanyon, L.E. (2008) Mechanical Loading Enhances the Anabolic Effects of Intermittent Parathyroid Hormone (1-34) on Trabecular and Cortical Bone in Mice. Bone, 43, 238-248. https://doi.org/10.1016/j.bone.2008.04.012

[11] Chow, J.W., Fox, S., Jagger, C.J. and Chambers, T.J. (1998) Role for Parathyroid Hormone in Mechanical Responsiveness of Rat Bone. American Journal of Physiology, 274, E146-E154. https://doi.org/10.1152/ajpendo.1998.274.1.E146

[12] Shimada, Y., Sakuraba, T., Matsunaga, T., Misawa, A., Kawatani, M. and Itoi, E. (2006) Effects of Therapeutic Magnetic Stimulation on Acute Muscle Atrophy in Rats after Hindlimb Suspension. Biomedical Research, 27, 23-27. https://doi.org/10.2220/biomedres.27.23

[13] Takakura, A., Lee, J.W., Hirano, K., Isogai, Y., Ishizuya, T., Takano-Kawabata, R. and Iimura, T. (2017) Administration Frequency as Well as Dosage of PTH Are Associated with Development of Cortical Porosity in Ovariectomized Rats. Bone Research, 5, Article No. 17002. https://doi.org/10.1038/boneres.2017.2

[14] Akagawa, M., Miyakoshi, N., Kasukawa, Y., Ono, Y., Yuasa, Y., Nagahata, I., Sato, C., Tsuchie, H., Nagasawa, H., Hongo, M. and Shimada, Y. (2018) Effects of Activated Vitamin D, Alfacalcidol, and Low-Intensity Aerobic Exercise on Osteopenia and Muscle Atrophy in Type 2 Diabetes Mellitus Model Rats. PLoS ONE, 13, e0204857. https://doi.org/10.1371/journal.pone.0204857

[15] Laib, A., Kumer, J.L., Majumdar, S. and Lane, N.E. (2001) The Temporal Changes of Trabecular Architecture in Ovariectomized Rats Assessed by MicroCT. Osteoporosis International, 12, 936-941. https://doi.org/10.1007/s001980170022

[16] Murata, K. and Yano, E. (2002) Medical Statistics for Evidence-Based Medicine with SPBS User's Guide. Nankodo, Tokyo.

[17] Sornay-Rendu, E., Boutroy, S., Duboeuf, F. and Chapurlat, R.D. (2017) Bone Microarchitecture Assessed by HR-pQCT as Predictor of Fracture Risk in Postmenopausal Women: The OFELY Study. Journal of Bone and Mineral Research, 32, 1243-1251. https://doi.org/10.1002/jbmr.3105 
[18] Washimi, Y., Ito, M., Morishima, Y., Taguma, K., Ojima, Y., Uzawa, T. and Hori, M. (2007) Effect of Combined Human PTH (1-34) and Calcitonin Treatment in Ovariectomized Rats. Bone, 41, 786-793. https://doi.org/10.1016/j.bone.2007.06.019

[19] Brouwers, J.E., van Rietbergen, B., Huiskes, R. and Ito, K. (2009) Effects of PTH Treatment on Tibial Bone of Ovariectomized Rats Assessed by in Vivo Micro-CT. Osteoporosis International, 20, 1823-1835.

https://doi.org/10.1007/s00198-009-0882-5

[20] Miki, T., Nakatsuka, K., Naka, H., Masaki, H., Imanishi, Y., Ito, M., Inaba, M., Morii, H. and Nishizawa, Y. (2004) Effect and Safety of Intermittent Weekly Administration of Human Parathyroid Hormone 1-34 in Patients with Primary Osteoporosis Evaluated by Histomorphometry and Microstructural Analysis of Iliac Trabecular Bone before and after 1 Year of Treatment. Journal of Bone and Mineral Metabolism, 22, 569-576. https://doi.org/10.1007/s00774-004-0525-Z

[21] Lane, N.E., Thompson, J.M., Strewler, G.J. and Kinney, J.H. (1995) Intermittent Treatment with Human Parathyroid Hormone (hPTH[1-34]) Increased Trabecular Bone Volume But Not Connectivity in Osteopenic Rats. Journal of Bone and Mineral Research, 10, 1470-1477. https://doi.org/10.1002/jbmr.5650101007

[22] Notomi, T., Lee, S.J., Okimoto, N., Okazaki, Y., Takamoto, T., Nakamura, T. and Suzuki, M. (2000) Effects of Resistance Exercise Training on Mass, Strength, and Turnover of Bone in Growing Rats. European Journal of Applied Physiology, 82, 268-274. https://doi.org/10.1007/s004210000195

[23] Umemura, Y., Ishiko, T., Yamauchi, T., Kurono, M. and Mashiko, S. (1997) Five Jumps per Day Increase Bone Mass and Breaking Force in Rats. Journal of Bone and Mineral Research, 12, 1480-1485. https://doi.org/10.1359/jbmr.1997.12.9.1480

[24] Welch, J.M., Weaver, C.M. and Turner, C.H. (2004) Adaptations to Free-Fall Impact Are Different in the Shafts and Bone Ends of Rat Forelimbs. Journal of Applied Physiology (1985), 97, 1859-1865. https://doi.org/10.1152/japplphysiol.00438.2004

[25] Hamann, N., Kohler, T., Müller, R., Brüggemann, G.P. and Niehoff, A. (2012) The Effect of Level and Downhill Running on Cortical and Trabecular Bone in Growing Rats. Calcified Tissue International, 90, 429-437. https://doi.org/10.1007/s00223-012-9593-6

[26] Cunningham, H.C., West, D.W.D., Baehr, L.M., Tarke, F.D., Baar, K., Bodine, S.C. and Christiansen, B.A. (2018) Age-Dependent Bone Loss and Recovery during Hindlimb Unloading and Subsequent Reloading in Rats. BMC Musculoskeletal Disorders, 19, 223. https://doi.org/10.1186/s12891-018-2156-X

[27] Gardinier, J.D., Mohamed, F. and Kohn, D.H. (2015) PTH Signaling during Exercise Contributes to Bone Adaptation. Journal of Bone and Mineral Research, 30, 1053-1063. https://doi.org/10.1002/jbmr.2432 